\title{
Cannabis sativa L. oil extract affects neuroinflammation, clinical score, and cannabinoid receptor-1 gene expression in $\mathrm{C} 57 \mathrm{bl} / 6$ experimental autoimmune encephalomyelitis
}

\author{
Iman Goraki ${ }^{1 *}{ }^{\circledR}$, Mohammad Reza Shiri-Shahsavar ${ }^{2,3 *}{ }^{\circledR}$, Mohammad Pourhassan-Moghaddam $^{4}{ }^{\circledR}$, Sharareh Khangaldi ${ }^{5^{\circledR}}$, \\ Sina Khodakarimi ${ }^{6,9}{ }^{\circledR}$, Mohammad Ebrahimi-kalan $^{7 \odot}$, Mohammad Jafar Maleki $^{\circledR}{ }^{\circledR}$, Hanieh Beyrampour-Basmenj ${ }^{8,9}{ }^{\circledR}$, Abbas $^{\circ}$ \\ Ebrahimi-kalan $^{6 * \mathbb{C}}$ \\ ${ }^{1}$ Department of Molecular Biology, Islamic Azad University Ahar Branch, Tabriz, Iran \\ ${ }^{2}$ Department of Nutrition, School of Health, Qazvin University of Medical Sciences, Qazvin, Iran \\ ${ }^{3}$ Cellular and Molecular Research Center, Research Institute for Prevention of Non-Communicable Diseases, Qazvin University of Medical \\ Sciences, Qazvin, Iran \\ ${ }^{4}$ Institute for Biomedical Materials and Devices (IBMD), Faculty of Science, University of Technology Sydney, Sydney, NSW 2007, Australia \\ ${ }^{5}$ Department of Nutrition, School of Nutrition, Tabriz University of Medical Sciences, Tabriz, Iran \\ ${ }^{6}$ Department of Neurosciences and cognition, School of Advanced Medical Sciences, Tabriz University of Medical Sciences, Tabriz, Iran \\ ${ }^{7}$ Department of Epidemiology, Stempel College of Public Health, Florida International University, Miami, FL, USA \\ ${ }^{8}$ Department of Medical Biotechnology, School of Advanced Medical Sciences, Tabriz University of Medical Sciences, Tabriz, Iran \\ ${ }^{9}$ Student Research Committee, Tabriz University of Medical Sciences, Tabriz, Iran
}

*Correspondence to

Abbas Ebrahimi-kalan; Email:

Ebrahimiab@tbzmed.ac.ir,

Abbasebra@gmail.com

\# Iman Goraki and

Mohammad Reza Shiri-

Shahsavar contributed equally

to this work.

Received 1 May 2021

Accepted 16 June 202

Published online 31 July 2021

Citation: Goraki I, Shiri-

Shahsavar M, Pourhassan-

Moghaddam M,

Khangaldi S, Khodakarimi

S, Ebrahimi-kalan M,

Maleki M, Beyrampour-

Basmenj H, Ebrahimi-

kalan A. Cannabis sativa

L. oil extract affects

neuroinflammation,

clinical score, and

cannabinoid receptor-1

gene expression in $\mathrm{C} 57 \mathrm{bl} / 6$

experimental autoimmune

encephalomyelitis.

Immunopathol Persa.

2022;8(1):e04.

DOI:10.34172/

ipp.2022.04.

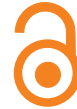

\section{Abstract}

Introduction: Multiple sclerosis (MS) is a degenerative central nervous system disease derived by immune mechanisms, which ultimately results in clinical debilities. Numerous nutraceuticals have been cited to be effective in treatment of central nervous system complications.

Objectives: This study investigated the effect of Cannabis sativa L. seed oil on experimental autoimmune encephalomyelitis (EAE).

Materials and Methods: Female C57bl/6 mice were assigned randomly into three groups ( 8 in each). Group-A received no myelin oligodendrocyte glycoprotein (MOG), group B was immunized by MOG and treated with oil, while in group $\mathrm{C}$ animals were immunized and treated with normal saline. Clinical scores were recorded every other day throughout the study and after four weeks, all mice were sacrificed and spinal cords were incised for molecular and histopathological evaluations.

Results: Significant differences were observed in mean clinical scores between control and experiment groups $(P<0.001)$. Cannabinoid receptor-1 gene expression increased significantly in treatment group $(P<0.001)$. Histopathologic evaluations also showed a significant decrease in overall infiltrated and vacuolated area and immune cells infiltration into the central nervous system in the treatment group $(P<0.01)$.

Conclusion: Cannabis sativa L. oil extract administration alleviated inflammation and paralysis in animal model. Therefore, its oil extract might be useful in soothing inflammatory and auto-immune diseases. However, additional research might be required.

Keywords: Cannabis sativa L. oil extract, Neuroinflammation, Histopathology, Cannabinoid receptor-1 gene expression, Multiple sclerosis, Experimental autoimmune encephalomyelitis

\section{Introduction}

Multiple sclerosis (MS) is a central nervous system (CNS) disease derived by immune dysfunction (1). The immune cells notably lymphocytes, infiltrate into CNS by unknown stimulus which influence the blood brain barrier (BBB) permeability and initiate inflammatory responses (1). Many demyelinating cell types identified in CNS lesions (mainly CD4 $4^{+}, \mathrm{CD}^{+} \mathrm{T}$ cells, monocyte derived macrophages, and microglia cells) which orchestrate an autoimmune response against myelin antigens (2). Owing to the immune cells invasion and their pivotal role in disease pathogenesis, MS was considered as an autoimmune disease. The lesions or plaques are generally in the white matter, targeting myelin sheet and vacuolization it in brain and spinal cord (2). Ultimately these complications manifest debilitating

Copyright $\odot 2022$ The Author(s); Published by Nickan Research Institute. This is an open-access article distributed under the terms of the Creative Commons Attribution License (http://creativecommons.org/licenses/by/4.0), which permits unrestricted use, distribution, and reproduction in any medium, provided the original work is properly cited. 


\section{Key point}

Nutraceutical components have been evaluated in treatment of Multiple sclerosis. This study designed to investigate the effects of Cannabis sativa L. seed oil on animal of multiple sclerosis and behavioral, molecular and histopathological consideration showed mentioned extract administration alleviated inflammation and paralysis in experimental autoimmune encephalomyelitis. Therefore, it might be useful in soothing inflammatory and auto-immune diseases.

clinical condition with serious consequences. It is a matter of concern, since MS is the most common cause of neurological disability with incidence rate of 2.0 men and 3.6 women per 100000 person-years (3).

Experimental autoimmune encephalomyelitis (EAE) is an animal model that widely used by researchers which resembles relapsing remitting MS and demonstrates similar indications in mice (4). In susceptible strains of mouse models, EAE is induced by active immunization using CNS homogenates. The procedure is composed of subcutaneous injections of myelin or myelin-derived antigens-for instance myelin oligodendrocyte glycoprotein (MOG) emulsified in adjuvant - that is preferably injected near lymph node surrounding areas like flanks. Antigen presenting cells mature and present myelin-derived peptides to naive $\mathrm{T}$ cells in the lymph nodes subsequent to immunization (4). Activation and differentiation of $\mathrm{T}$ cells particularly $\mathrm{T}$ helper-1 (Th1) and T helper-17 (Th17) cells, proceeded to upregulation and secretion of proinflammatory cytokines, notably interferon-gamma $($ IFN- $\gamma$ ) have been shown to play pivotal role in EAE induction (5).

However, the role of different $\mathrm{T}$ helper subtypes has not been clarified in human studies as well as EAE. T regulatory (Treg) cells possess suppressor function and a defect in immune-tolerance mechanism or effector cells, may preclude the suppression by Tregs both in MS and EAE (5). Recently explored endocannabinoid system revealed regulatory function of numerous endogenous ligands, enzymes and receptors, cooperating in immune and nervous system (6). Although two main cannabinoid receptors $(\mathrm{CB}) 1$ and 2 had been identified so far, there are possibilities of existing other receptors and agents involved in this mediatory system (7). Currently, it has been substantiated that CB1 - expressed in CNS mediates neuroprotection in CNS, while CB2 - mostly expressed in immune cells - regulates immune responses (8). These receptors can be activated by different types of either natural or synthetic cannabinoid derivatives. Hence, it is hypothesized that the neuroprotective attribute of cannabinoids come from CB1 function (9). Lately, Cannabis sativa L. and its oil have received growing interests due largely to its nutritional and traditional herbal values that are associated with health benefits. C. sativa seed oil (CSO) is nutritionally well balanced and considered as a valuable source of important fatty acids, mainly omega 3 alpha-linolenic acid (ALN; 18:3n3) and omega 6 linoleic acid (LA; 18:2n6) (10). The optimal nutritional ratio of LA to ALN is 3:1 which is desirably pursued in CSO. These two polyunsaturated fatty acids (PUFAs) possess potential anti-inflammatory, antithrombotic and antiarrhythmic attributes (11).

Moreover, presence of gamma-linolenic acid (GLA; 18:3n6) in CSO makes it preferable to other comparable seed oils. It is also one of the few botanical sources of GLA and stearidonic acid (18:4n3) (12). The fatty acid composition of CSO and its pharmacologically active constituents have gained much attention recently. CSO also have noteworthy volumes of tocopherols and other terpenoids, which are stated to display vital antioxidant, anticancer, antiinflammatory, and anti-thrombotic activity along with omega-3 PUFAs (11). It is claimed that the anticonvulsive, anti-epileptic, and antimicrobial features of CSO is due to the presence of cannabidiol (CBD), although the CBD concentrations may seem low within the oil. Both CBD and delta-9-tetrahydrocan-nabinol (THC) production mostly happens in the glandular structures of the plant, though the presence of these two major cannabinoids is a result of contamination during the course of pressing or extraction (12). Various cannabinoid derivative has been identified so far, but the quantity of CBD in different cannabis varieties is generally higher than THC, therefore as expected, the $\mathrm{CBD}$ contamination and/or concentration in this oil is greater than that of THC.

\section{Objectives}

Myriad of drugs and compounds have been introduced for this neuro-inflammatory condition. However, none is able to cure this disease solely. Accordingly, nutritional approaches employ numerous nutraceuticals simultaneously in treatment of this disease. In this study, we have used the cold pressed extract of Cannabis sativa L. seed oil to observe whether the EAE symptoms, inflammation and CB1 gene expression would be altered or not.

\section{Materials and Methods \\ Experimental mice}

In this study 24 female mice (race C57bl/6; age 9 weeks; average weight $20 \pm 2 \mathrm{~g}$ ) were provided from Pastor Institute (Iran) and randomly divided into three groups: healthy group (A), CSO group (B) and control group (C). Mice were acclimatized and housed in animal house of neurosciences research center, Tabriz university of medical sciences (Tabriz, Iran) on 12/12 day/night cycles and sanitary condition.

\section{EAE induction}

Hooke kits MOG 35-55 (Hooke Laboratories, Lawrence, MA, USA) were utilized for subcutaneous induction of EAE based on the manufacturer's instruction. MOG3555/CFA emulsion was injected subcutaneously into both 
flanks (0.2 mL/animal), continued by 2 - and 24-hour intraperitoneal (i.p.) pertussis toxin $(0.1 \mathrm{~mL} / \mathrm{animal} / \mathrm{day}$, i.p.) injections.

\section{Oil extraction an administration}

Fresh C. sativa L. seed was purchased and its oil was extracted using cold press method. In group B, CSO (200 $\mu \mathrm{L} /$ mouse/day) was intraperitoneally injected every other day throughout the study. While in group $\mathrm{C}$, normal saline was injected i.p., following the same protocol.

\section{EAE evaluation}

Animals were evaluated daily for their clinical scores according to Table 1 . The clinical manifestation of EAE was diagnosed around day 10 post-immunization, with an acute condition after 3 to 5 days from onset of the clinical symptoms. This condition lasted 1 to 3 days and then clinical manifestation improved gradually in most cases. The severity of disease - with average daily scores of the mice in each group - was calculated and analyzed by repeated measure test, to compare the course of EAE between the two groups throughout the study.

\section{Spinal cord preparation}

Mice were perfused $4 \%$ paraformaldehyde solution in normal saline transcardially, to be assured of fixation. The spinal cord's tissue were dissected and immersed in paraformaldehyde $4 \%$, renewed once after two days, and reserved for histopathological evaluations.

\section{Histopathological evaluation}

The paraformaldehyde fixed spinal cord tissues were dehydrated, cleared, paraffinized and embedded in paraffin wax respectively and $5 \mu \mathrm{m}$ lumbar sections were provided with a rotary microtome (Leitz, Wetzlar, Germany). Samples were mounted on glass slides following the protocol for hematoxylin and eosin (H\&E) staining and subsequently, three sections of every spinal cord were evaluated under a light microscope (Axioskop2; Carl Zeiss MicroImaging Inc., Germany). Immune cells infiltration into spinal tissue was assessed using Infinity software 0.4.

\section{Molecular evaluation with real-time PCR}

Real-time PCR (SYBR Green) was used to detect CB1 expressions. Total RNA was isolated from the lumbar part of spinal cord using a EURX Gene MATRIX universal RNA purification kit, according to the recommended instruction. The purity and concentration of the isolated RNA were examined by a NanoDrop 2000c spectrophotometer (Thermo Fisher Scientific, Waltham, MA, USA). Reverse transcription of extracted RNA samples (up to $500 \mathrm{ng} /$ sample) of spinal tissue to cDNA was performed using AccuPower RocketScript kit according to the protocols. Real-time PCR was performed in MIC real-time PCR system using SYBR Premix Ex TaqII (Takara Bio Inc., Japan) according to the manufacturer's protocol. Using GenScript's online real-time primer design tool, primers were designed and booked from Macrogen Inc (Table 2). The housekeeping gene was glyceraldehyde 3-phosphate dehydrogenase (GAPDH) for endogenous reference, and triplet reactions were carried out. The relative rate of distinct mRNA expression was measured by "Fold change $=2(-\Delta \Delta \mathrm{ct})$ " formula.

\section{Statistical analysis}

Data were reported with median (Q1-Q3) for nonparametric and mean \pm standard error for parametric analysis. The differences in mean score between two groups were measured and analyzed by Linear mixed model test and compared through the entire duration of study. SPSS (version 21) and MLwiN (version 10) were used to analyze data and $P$ values less than 0.05 were determined significant.

\section{Results}

\section{Clinical score}

In group $\mathrm{C}$, the first clinical symptoms developed on day 11, while on day 17 post-immunization all animals were totally afflicted. However, in group B, the first clinical score initiated on day 14 (Figure 1). The acute phase of EAE in group $C$ was observed on day 19 , however, in group $B$ the peak of disease delayed until day 23 and the mean clinical scores diminished from 2 to 1 (approximately $50 \%)$. Furthermore, mean clinical scores of the treatment and control groups were compared by linear mixed model analysis throughout the course of study and significant reduction was observed $(P<0.001$; Table 3$)$.

\section{Lymphocyte infiltration into the spinal cord}

Histopathological evaluation in the H\&E stained transverse lumbar sections of the mice demonstrated immune cells infiltration in the region adjacent to the anterior spinal cord artery in the anterior and posterior

Table 1. Clinical score of EAE

\begin{tabular}{ll}
\hline Score & Scale \\
\hline 0 & Normal \\
1 & Limp tail \\
2 & Hind limb weakness \\
\hline 3 & Hind limb paralysis \\
\hline 4 & Forelimb paralysis \\
\hline 5 & Moribund or death \\
\hline
\end{tabular}

Table 2. Primers sequence

\begin{tabular}{llll}
\hline Primer & Sequence $\left(\mathbf{5}^{\prime} \rightarrow \mathbf{3}^{\prime}\right)$ & Length & CG\% \\
\hline CB1 $(\mathrm{F})$ & ACTCAGACTGCCTGCACAAG & 20 & 55.00 \\
CB1 $(\mathrm{R})$ & TGGCGATCTTAACAGTGCTC & 20 & 50.00 \\
GAPDH $(\mathrm{F})$ & CACTGCCACCCAGAAGACTG & 20 & 60.00 \\
GAPDH $(\mathrm{R})$ & CCAGTGAGCTTCCCGTTCAG & 20 & 60.00 \\
\hline
\end{tabular}




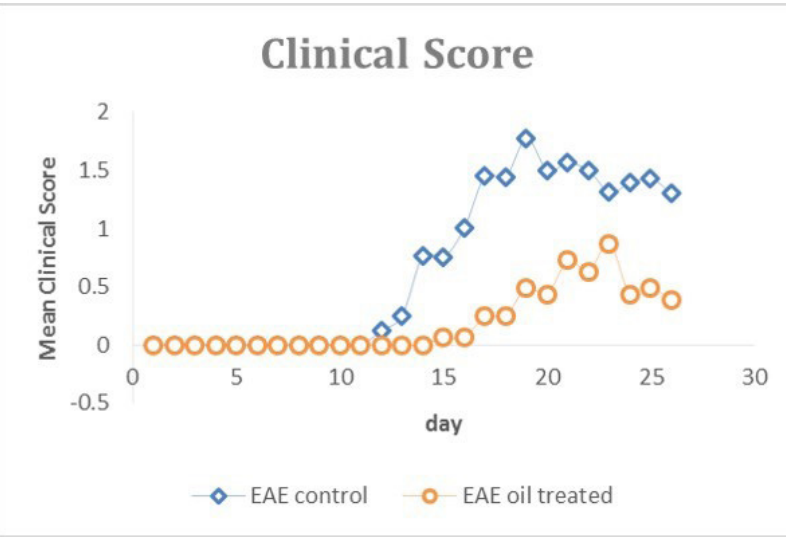

Figure 1. Mean clinical score in oil treated group was significantly lower compared to EAE control $(P<0.001)$.

funiculi (Figure 2). Histological assessment of sections showed that infiltrated area percentage in group B was significantly lower than group C (24.91 \pm 12.13 compared to $8.56 \pm 6.91 ; P=0.039$ ). These results suggest that CSO noticeably alleviated the clinical symptoms of EAE.

\section{Expression of CB1 in the lumbar spinal cord tissue}

Regulation of specific genes in neuroinflammatory condition such as EAE has been disorganized. Cannabinoid receptors are believed to be downregulated in neuroinflammatory disorders. Real-time PCR data indicated a significant up-regulation of CB1 gene expression in treatment group compared to the control group $(P<0.001$; Table 4$)$. A 2.05 -fold upregulation was detected in the experiment group in comparison to control (Figure 3).

\section{Discussion}

Cannabis sativa contains a wide range of nutrients and nutraceuticals with medicinal properties (13). Recently, scientists have expressed particular interest on its immune and neuro-modulatory properties due to its bioactive components like cannabinoids, vitamins, essential fatty acids and antioxidants. The potential neuro-protective features of these components have been cited in many researches (14). Neurodegeneration occurs in EAE by immune cells activation and their invasion into CNS in the first place. It is then followed by inflammatory and autoimmune reactions (15). Prominent proinflammatory biomarkers including IFN- $\gamma$, TNF-a, IL-1b and IL- 6 which are produced by immune cells were reduced in EAE upon treatment with $C$. sativa or its constituents (16). This

Table 3. The average scores in experimental groups

\begin{tabular}{lccc}
\hline \multirow{2}{*}{ Response } & \multicolumn{3}{c}{ Average score } \\
\cline { 2 - 4 } & Coefficient & SE & P value \\
\hline Constant & 0.678 & 0.091 & $<0.001$ \\
B vs. C & -0.467 & 0.058 & \\
\hline
\end{tabular}

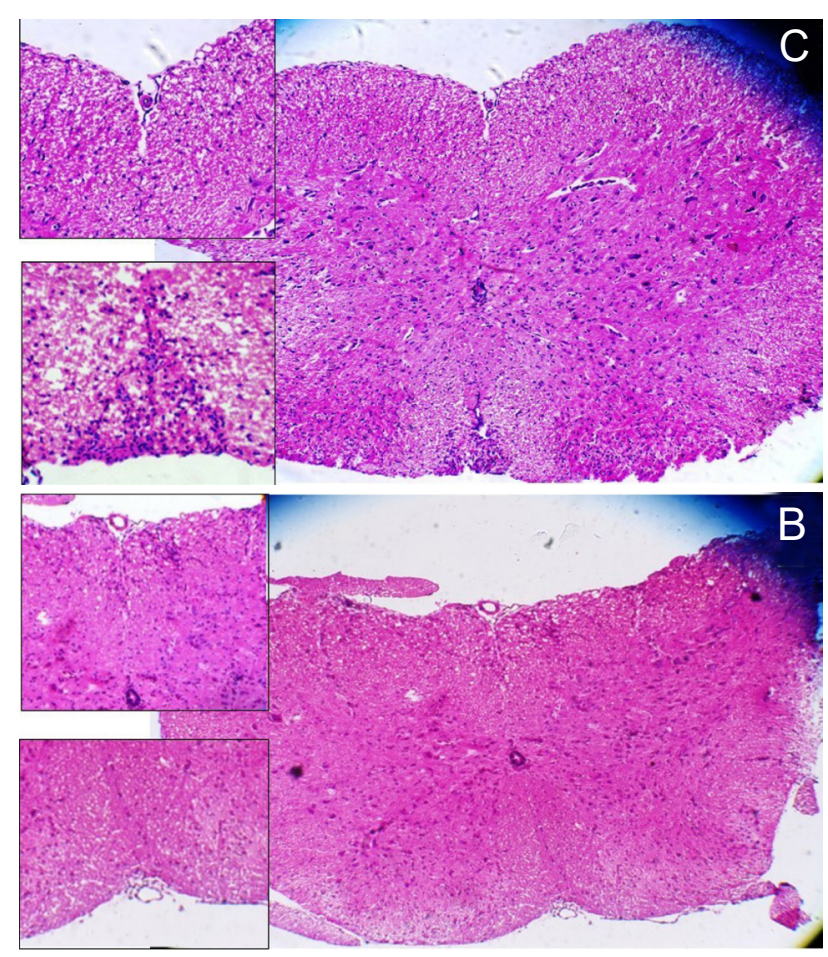

Figure 2. Inflammation in spinal cord sections. Infiltrated area percentage in group B was significantly lower than group C $(24.91 \pm 12.13$ compared to $8.56 \pm 6.91 ; P=0.039)$.

reveals the considerable potential of cannabis-derived products on neuroinflammation and neurodegeneration relief in MS patients (17). In this study, cold pressed $C$. sativa L. oil extract was used to evaluate its beneficiary effects on the related gene expression in molecular level and neuroinflammation and neurodegeneration in clinical level using molecular or histological assessments. As illustrated in Figure 1, an increase in mean clinical score was noted in EAE group after immunization with MOG, as debilities advanced simultaneously throughout the study $(P<0.001)$. During this study, CSO injection in experiment group significantly reduced the mean clinical score $(P<0.01)$ and debilitating signs of EAE when compared to the control group. Histological assessments demonstrated that induction and advancement of neurodegeneration in mice model of MS were concurrent with immune cell infiltration into the CNS, demyelination, vacuolization and general damage to neuronal function (18). Hematoxylin and eosin method in Figure 2 depicts the lumbar section of spinal cord and immune cells invasion of neural tube in control and experiment groups. CSO considerably reduced $(P<0.01)$ the number of immune cells painted

Table 4. CB1 expression fold change between group B and C

\begin{tabular}{lccc}
\hline & $\begin{array}{c}\text { CB1 expression } \\
(\text { mean } \pm \text { SD) }\end{array}$ & Fold change & P value \\
\hline Group B (Experiment) & $3.1 \pm 0.141$ & & \\
Group C (Control) & $1.5 \pm 0.141$ & 2.05 & $<0.01$ \\
\hline
\end{tabular}




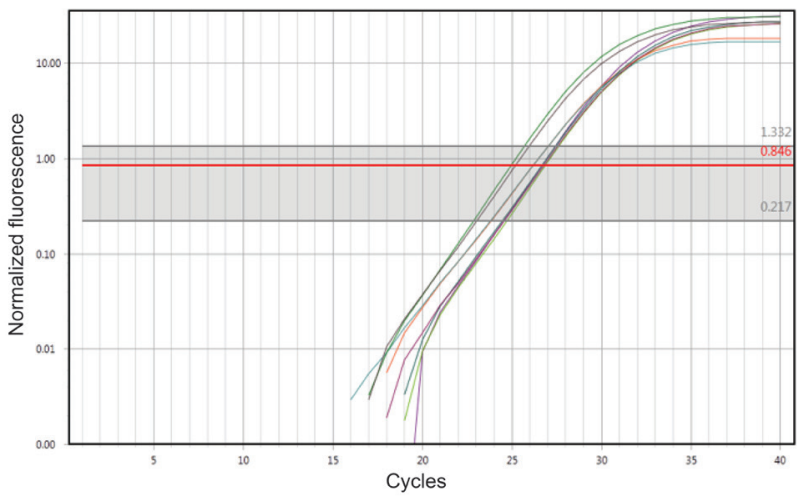

Expression level of CB1 gene

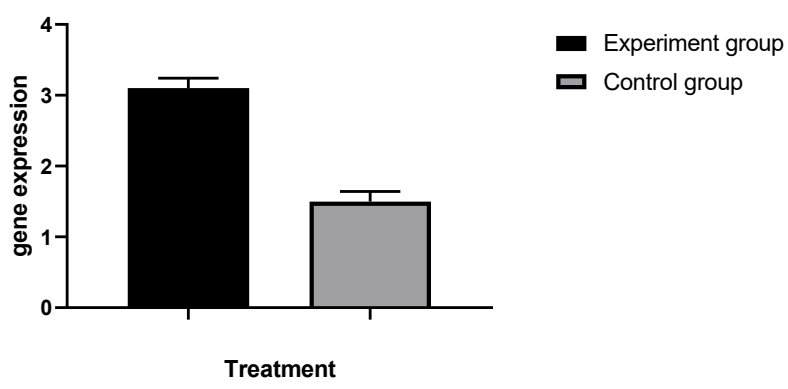

Figure 3. CB1 expression. Real-time PCR data indicated a significant upregulation of $\mathrm{CB} 1$ gene expression in the experiment group compared to the control group $(P$ value $<0.01)$.

and presented in spinal cord tissue. Moreover, the mean infiltrated and affected area per $\mu \mathrm{m}^{2}$ of tissue (in percentage) was significantly less in oil treated mice in comparison to untreated ones $(P=0.02)$. These findings suggest that immuno-nutrients including omega 6 PUFAs and omega 3 , essential fatty acids, antioxidants and vitamin E presence in CSO could possibly suppress neuroinflammation and immune cell activation and invasion, in line with other studies (19). It has been demonstrated that cannabinoids perform most of their actions via CB1 or CB2 (20) whereby, the neuroprotective properties are believed to be mediated mostly by CB1 (21). Studies show more rapid neurodegeneration progress during EAE in $\mathrm{CB} 1$ deficient mice, substantiating a CB1 receptor-dependent mechanism for neuroprotection. Numerous studies on EAE have recently reveals anti-inflammatory and antioxidant effects of CBD i.p. administration, alone or in combination with other compounds (21). CBD, the most abundant nonpsychoactive cannabinoid, has been detected in oil extract of $C$. sativa seed along with a large number of natural cannabinoids (22). As demonstrated in Figure 2, this study evaluated the expression of $\mathrm{CB} 1$ in CNS of EAE mice. The findings suggest that the expression of CB1 increased significantly in EAE mice treated with oil extract compared to EAE control $(P<0.001)$. The reduction of immune cell infiltration and decreased clinical debilities along with increased CB1 expression, confirm the neuroprotective activity of CB1, as well as neuroinflammatory suppressive properties of CSO. However, a probable overlap in addition to non-CB1/CB2-mediated mechanisms might have been premised.

\section{Conclusion}

In conclusion, innumerable evidence support cannabinoid compounds application in MS treatment through endocannabinoid signaling system, which offers a novel strategy in attenuating the disease advancement (23). Furthermore, the presence of immunoregulatory vitamins and antioxidants in CSO assists the restriction of neuroinflammation in treatment process (24). Additionally, essential PUFAs, which myriad of benefits have been attributed to their immunosuppressive role, might upregulate the expression of some certain neurological mediators (25).

\section{Acknowledgments}

We would also like to appreciate all colleagues that played a role and shared their pearls of wisdom throughout the course of this study.

\section{Authors' contribution}

IG and MSHSH were the principal investigators of the research. AEK and MPM were included in preparing and designing the concept. MEK revisited the manuscript and critically evaluated the intellectual contents. MJM and HBB collaborated in molecular part and SK help us in histopathological section. All authors participated in preparing the final draft of the manuscript, revised the manuscript and critically evaluated the intellectual contents. All authors have read and approved the content of the manuscript and confirmed the accuracy or integrity of any part of the work.

\section{Conflicts of interest}

The authors declare no conflicts of interest.

\section{Ethical issues}

This study was approved by Ethics Committee of Islamic Azad University, Ahar branch (Ethical code\# 22030503932027). This investigation was also conducted according to the regulations of the Research Ethics Committee of Iranian Ethical Guidelines for the use of animals in research. Additionally, all animal experiments were in accordance with protocols approved by the United States National Institutes of Health $(\mathrm{NIH}, 1978)$. This study was extracted from MSc thesis of Iman Goraki at this university (Thesis \#22030503932027). Moreover, ethical issues (including plagiarism, data fabrication, double publication) have been completely observed by the authors.

\section{Funding/Support}

Supportive funding of Iran National Science Foundation (96013435) was used additionally for the development of this research too.

\section{References}

1. Dendrou CA, Fugger L, Friese MA. Immunopathology of multiple sclerosis. Nat Rev Immunol. 2015;15:545-58. doi: 10.1038/nri3871.

2. Steinman L. Immunology of relapse and remission in multiple sclerosis. Annu Rev Immunol. 2014;32:257-81. doi: 10.1146/ annurev-immunol-032713-120227.

3. Leray E, Moreau T, Fromont A, Edan G. Epidemiology of multiple sclerosis. Rev Neurol (Paris). 2016;172:3-13. doi: 10.1016/j.neurol.2015.10.006. 
4. Mix E, Meyer-Rienecker H, Zettl UK. Animal models of multiple sclerosis for the development and validation of novel therapies - potential and limitations. J Neurol. 2008;255 Suppl 6:7-14. doi: 10.1007/s00415-008-6003-0.

5. Hedegaard CJ, Krakauer M, Bendtzen K, Lund H, Sellebjerg F, Nielsen $\mathrm{CH}$. T helper cell type 1 (Th1), Th2 and Th17 responses to myelin basic protein and disease activity in multiple sclerosis. Immunology. 2008;125:161-9. doi: 10.1111/j.13652567.2008.02837.

6. Kim J, Carlson ME, Kuchel GA, Newman JW, Watkins BA. Dietary DHA reduces downstream endocannabinoid and inflammatory gene expression and epididymal fat mass while improving aspects of glucose use in muscle in C57BL/6J mice. Int J Obes (Lond). 2016;40:129-37. doi: 10.1038/ijo.2015.135.

7. Berglund BA, Fleming PR, Rice KC, Shim JY, Welsh WJ, Howlett AC. Development of a novel class of monocyclic and bicyclic alkyl amides that exhibit CB1 and CB2 cannabinoid receptor affinity and receptor activation. Drug Des Discov. 2000;16:281-94.

8. Jean-Gilles L, Braitch M, Latif ML, Aram J, Fahey AJ, Edwards LJ, et al. Effects of pro-inflammatory cytokines on cannabinoid CB1 and CB2 receptors in immune cells. Acta Physiol (Oxf). 2015;214:63-74. doi: 10.1111/apha.12474.

9. Docagne F, Muñetón V, Clemente D, Ali C, Loría F, Correa F, et al. Excitotoxicity in a chronic model of multiple sclerosis: Neuroprotective effects of cannabinoids through CB1 and CB2 receptor activation. Mol Cell Neurosci. 2007;34:551-61. doi: 10.1016/j.mcn.2006.12.005.

10. Montserrat-de la Paz S, Marín-Aguilar F, García-Giménez MD, Fernández-Arche MA. Hemp (Cannabis sativa L.) seed oil: analytical and phytochemical characterization of the unsaponifiable fraction. J Agric Food Chem. 2014;62:110510. doi: 10.1021/jf404278q.

11. Smeriglio A, Galati EM, Monforte MT, Lanuzza F, D'Angelo V, Circosta C. Polyphenolic compounds and antioxidant activity of cold-pressed seed oil from Finola cultivar of Cannabis sativa L. Phytother Res. 2016;30:1298-307. doi: 10.1002/ptr.5623.

12. Leizer C, Ribnicky D, Poulev A, Dushenkov S, Raskin I. The composition of hemp seed oil and its potential as an important source of nutrition. J Nutraceuticals, Funct Med foods. 2000;2:35-53. doi: 10.1300/J133v02n04_04.

13. Da Porto C, Decorti D, Natolino A. Potential oil yield, fatty acid composition, and oxidation stability of the hempseed oil from four Cannabis sativa L. cultivars. J Diet Suppl. 2015;12:110. doi: 10.3109/19390211.2014.887601.

14. Girgih AT, Alashi AM, He R, Malomo SA, Raj P, Netticadan T, Aluko RE. A novel hemp seed meal protein hydrolysate reduces oxidative stress factors in spontaneously hypertensive rats. Nutrients. 2014;6:5652-66. doi: 10.3390/nu6125652.

15. Sajad M, Zargan J, Chawla R, Umar S, Sadaqat M, Khan
HA. Hippocampal neurodegeneration in experimental autoimmune encephalomyelitis (EAE): potential role of inflammation activated myeloperoxidase. Mol Cell Biochem. 2009;328:183-8. doi: 10.1007/s11010-009-0088-3.

16. Giacoppo S, Soundara Rajan T, Galuppo M, Pollastro F, Grassi G, Bramanti P, Mazzon E. Purified Cannabidiol, the main non-psychotropic component of Cannabis sativa, alone, counteracts neuronal apoptosis in experimental multiple sclerosis. Eur Rev Med Pharmacol Sci. 2015;19:4906-19.

17. Izzo AA, Capasso R, Aviello G, Borrelli F, Romano B, Piscitelli $F$, et al. Inhibitory effect of cannabichromene, a major nonpsychotropic cannabinoid extracted from Cannabis sativa, on inflammation-induced hypermotility in mice. Br J Pharmacol. 2012;166:1444-60. doi: 10.1111/j.1476-5381.2012.01879.

18. Fagone P, Mangano K, Quattrocchi C, Motterlini R, Di Marco R, Magro G, et al. Prevention of clinical and histological signs of proteolipid protein (PLP)-induced experimental allergic encephalomyelitis (EAE) in mice by the water-soluble carbon monoxide-releasing molecule (CORM)-A1. Clin Exp Immunol. 2011;163:368-74. doi: 10.1111/j.1365-2249.2010.04303.

19. van Meeteren ME, Teunissen CE, Dijkstra CD, van Tol EA. Antioxidants and polyunsaturated fatty acids in multiple sclerosis. Eur J Clin Nutr. 2005;59:1347-61. doi: 10.1038/ sj.ejcn. 1602255

20. Onaivi ES, Ishiguro H, Gu S, Liu QR. CNS effects of CB2 cannabinoid receptors: beyond neuro-immuno-cannabinoid activity. J Psychopharmacol. 2012;26:92-103. doi: 10.1177/0269881111400652.

21. Maresz K, Pryce G, Ponomarev ED, Marsicano G, Croxford JL, Shriver LP, et al. Direct suppression of CNS autoimmune inflammation via the cannabinoid receptor CB1 on neurons and CB2 on autoreactive T cells. Nat Med. 2007;13:492-7. doi: 10.1038/nm1561.

22. Hanus L, Subová D. The amount of main cannabinoid substances in hemp, cultivated for industrial fibre production and their changes in the course of one vegetation period. Acta Univ Palacki Olomuc Fac Med. 1989;122:11-23.

23. Zhang M, Martin BR, Adler MW, Razdan RJ, Kong W, Ganea $\mathrm{D}$, et al. Modulation of cannabinoid receptor activation as a neuroprotective strategy for EAE and stroke. J Neuroimmune Pharmacol. 2009;4:249-59. doi: 10.1007/s11481-009-91484.

24. Besler HT, Comoğlu S, Okçu Z. Serum levels of antioxidant vitamins and lipid peroxidation in multiple sclerosis. Nutr Neurosci. 2002;5:215-20. doi:10.1080/10284150290029205.

25. Mehta LR, Dworkin RH, Schwid SR. Polyunsaturated fatty acids and their potential therapeutic role in multiple sclerosis. Nat Clin Pract Neurol. 2009;5:82-92. doi: 10.1038/ ncpneuro1009. 